\title{
REMOTE SENSING AND CROWD-SOURCING
}

\author{
Raffaella Guida, Peter T.B. Brett and Salman S. Khan \\ Surrey Space Centre, University of Surrey \\ Guildford, United Kingdom \\ r.guida@surrey.ac.uk
}

\begin{abstract}
Collection of ground truth to validate remote sensing classification and/or detection algorithms is rarely accounted for due to the inaccessibility of the sites or the elevated costs of such operations.

In this paper some of the opportunities behind crowdsourcing are explored through the description of a remote sensing project on water quality monitoring in Africa where the ground truth was collected involving and training people from local communities.
\end{abstract}

Index Terms - water quality, crowd-sourcing.

\section{INTRODUCTION}

The collection and/or the availability of ground truth is still one of the main drawbacks in several remote sensing studies in which, very often, new models and methodologies are introduced and widely discussed but tested on either simulated data with obvious limitations or on real data without ground truth.

Crowd-sourcing is very recently emerging as an approach, applicable to any discipline, to collectively look at a common problem, solve it and share the solution.

In this paper a crowd-sourcing approach is applied to an environmental and social problem partly investigated with remote sensing data.

More specifically, the paper contains the outcomes of a multidisciplinary project run at the University of Surrey with the aim of monitoring the incidence of water-related diseases like diarrhoea in Malawi (Africa) by correlating remote sensing data acquired on inland waters and local information such as daily school attendance of students.

The paper is organized as follows: in Section 2 an overview of the project is given; Section 3 contains the remote sensing data processing and some preliminary results. Finally conclusions are drawn.

\section{PROJECT'S OVERVIEW}

Most of Malawi's 11 million people live in rural areas where access to safe water is very low (only 40\%), waterrelated diseases are widespread and child deaths (under five) from diarrhoea per annum are 9100 [1].

Lake Chilwa, in the south-west region of Malawi, represents an important catchment area for local people and more information on the quality of its water over different seasons is desired to understand the incidence of diarrhoea in children.

In the summer 2012 a multidisciplinary team made of engineers, environmental lawyers, biologists and psychologists based at the University of Surrey was formed to work at the problem of water quality monitoring in Malawi with remote sensing data. An important aspect of the project was to involve the local communities, in particular children, parents and teachers, in the collection of local information. Two were the kinds of information required and to be correlated with the outcomes of the remote sensing data processing: that on daily hygiene habits and health conditions of pupils and that on the water quality of Lake Chilwa.

The collection of this information was organized by the team but mainly conducted by locals and represents the crowd-sourcing experimental part of the project. The information on daily diseases in the schools was collected through an application for smartphones designed ad-hoc. The smartphones were purchased and set up in Surrey before being sent to Malawi. The application essentially contained a questionnaire, approved by an ethical commission, on hands hygiene and health of the children who were invited to enter their answers every day over the period of remote sensing data acquisition. The consensus of parents had to be required in this respect. Local teachers were trained in the use of the smartphones and the application by a member of the team who visited the schools involved at the beginning of the project. The teachers trained the students in turn.

Other locals were trained in the use of a DelAgua water testing kit to acquire and analyze samples of water in the lake in the proximity of the school. 
Both pieces of information are going to be processed and sent back to the team to allow a comparison with outcomes from remote sensing data processing which is described in the next section.

\section{DATA PROCESSING}

Different water quality parameters, such as suspended sediments and chlorophyll index, can be measured with remote sensing techniques [2].

In this project a particular interest is devoted to the possibility to detect algal blooms as sewage treatment and agricultural sources may cause an increase in nutrients in the lake sometimes resulting in intensive bloom of phytoplankton. Some species of phytoplankton produce toxins and are then harmful for human beings.

While recognizing different algal blooms is still a hard task, the detection of blooms with remote sensing techniques is quite straightforward [3] and in some cases the Normalized Difference Vegetation Index (NDVI), usually adopted to monitor the chlorophyll content in vegetation [4], has been applied to water bodies to detect algal blooms [5].

Similarly, NDVI maps have been produced in this study to detect a potential algal bloom in Lake Chilwa.

UK-DMC2 datasets from the Disaster Monitor Constellation have been acquired and processed on Lake Chilwa. With this sensor red, green and near-infrared channels are available at a spatial resolution of $22 \mathrm{~m}$. The acquisition campaign started on 25 th December and was completed on 31st January 2013. A total of six coverages, spaced every week, was ordered. Due to the intense cloud coverage in the rain season, only two datasets were considered suitable for this study. These datasets have been acquired on $3^{\text {rd }}$ and $17^{\text {th }}$ January 2013. In some areas clouds were still present but not in the regions of the lake in proximity of the schools and where water samples were collected. In the following we will refer to these images as image $A$ and $B$ respectively.

To make it possible to easily compare the values in the two images $A$ and $B$, ENVI software was used to coregister them and accurately line them up with each other. Due to the cloud cover in both images, the automatic registration tools were not able to find a usable set of control points (GCPs). Instead, we manually defined a set of 20 GCPs, using Map $\rightarrow$ Registration $\rightarrow$ Select GCPs: Image to Image. The estimated RMS error was 0.625 using polynomial transformation of degree 2. The GCPs manually defined were used to warp image $B$ so that it fitted image $A$ using Map $\rightarrow$ Registration $\rightarrow$ Warp from GCPs: Image to Image. The cubic convolution resampling mode was adopted. In order to make the comparison of the images easier, the warped image $B^{\prime}$ was made of the same size as image $A$. The coregistered images are shown in Figure 1.

The coregisterd images are rendered in false colour: the NIR channel is drawn in red, the red channel is drawn in green, and the green channel is drawn in blue.
The image products supplied by DMCii Ltd. encode the brightness in each band using 8 bits per pixel. However, the metadata supplied along with the products allow this digital number (DN) value to be converted to an explicit radiance value, according to the relation:

$$
\text { Radiance }=\frac{D N}{\text { Rescale Gain }}+\text { Rescale Bias }
$$

The Rescale Gain and the Rescale Bias were automatically loaded by the ENVI importer for DMC data.

Unfortunately, atmospheric correction could not be applied because the ENVI QUick Atmospheric Correction module was not available but this missing correction step has not affect the detection itself and quantitative analyses will not be performed at this stage considering the pilot nature of the project.

As next step, NDVI maps were produced.

The NDVI is defined as [4]:

$$
N D V I=\frac{N I R-R E D}{N I R+R E D}
$$

where NIR is the near infra-red band radiance, and RED is the red band radiance.

We calculated the index for each of the coregistered radiance images using ENVI's built-in Spectral $\rightarrow$ Vegetation Analysis $\rightarrow$ Vegetation Index Calculator tool.

A close-up view of NDVI images is given in Figure 2.

In both images the waters of Lake Chilwa presenting a high NDVI response extend over a very large area represented by grey/light grey pixels against the dark background of the lake. The shape in which the pixels cluster together clearly suggests an algal bloom. In two weeks time, the bloom has moved from south to north, decreased its extension and response to NDVI (shown by darker pixels in the processed image relevant to the dataset acquired on $17^{\text {th }}$ January, Fig.2(b)).

In the map relevant to the acquisition on $3^{\text {rd }}$ January the bloom is very close to the south-west side of the lake in the proximity of which the schools involved are situated. Also, the water samples were collected in the south region.

These preliminary results from remote sensing data processing will now be passed to the biologist in the team for comparison with the analysis of the water samples. The results from the water testing kit are now returning from Malawi and the next step will be to compare and correlate all the data collected, in situ or from remote.

In the meanwhile a visualization harness has been set up with the purpose to facilitate any comparison analysis and to work as a reference for local people to stay involved in the project and be informed about the outcomes. Finally the harness can be used to support decision-making processes. By the time of the conference the data integration and comparison will be completed and the final results will be presented and widely discussed. 


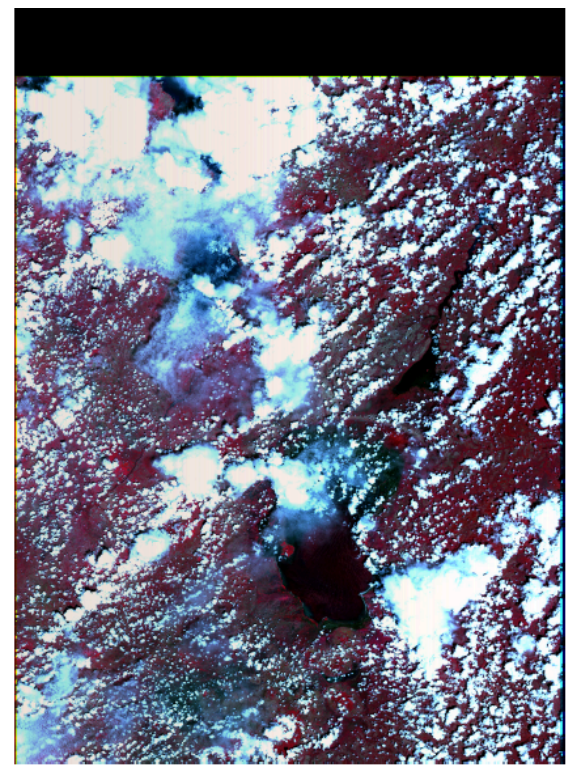

(a) 2013-01-03 (image $A$ )

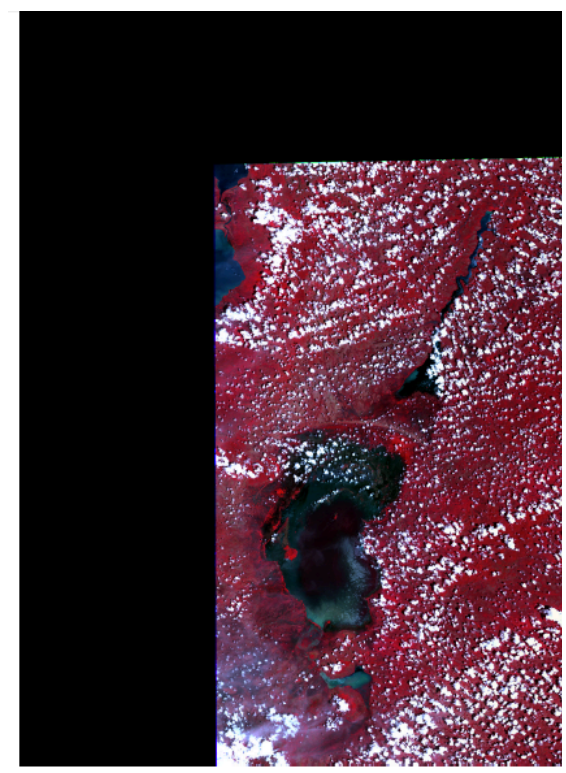

(b) 2013-01-17 (warped image $B$ )

Figure 1 - Coregistered images in false colour: (a) UKDMC2 acquisition of $3^{\text {rd }}$ January 2013; (b) UK-DMC2 acquisition of $17^{\text {th }}$ January 2013.

\section{CONCLUSIONS}

This paper presented preliminary results of a multidisciplinary project in Malawi with two main objectives: to monitor the water quality of its main water reservoir and, at the same time, the incidence of water-borne diseases in children. The project presented many challenges and, among these, the collection of ground truth through the engagement of local communities.

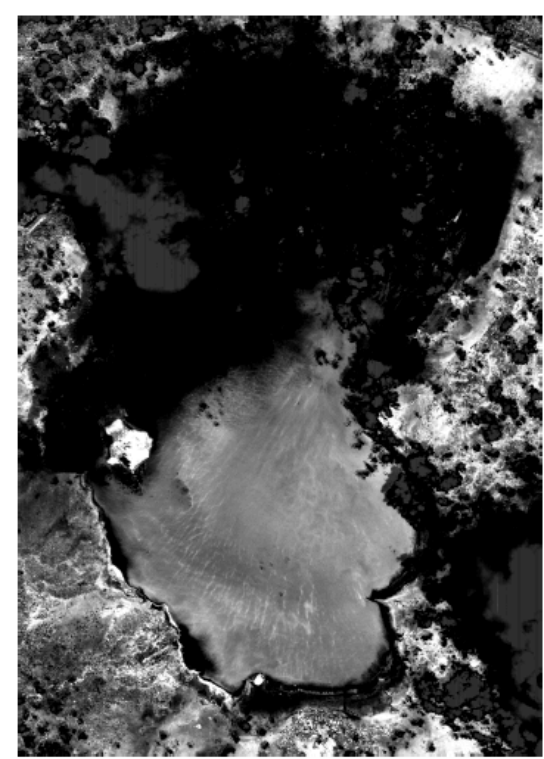

(a) 2013-01-03 NDVI map

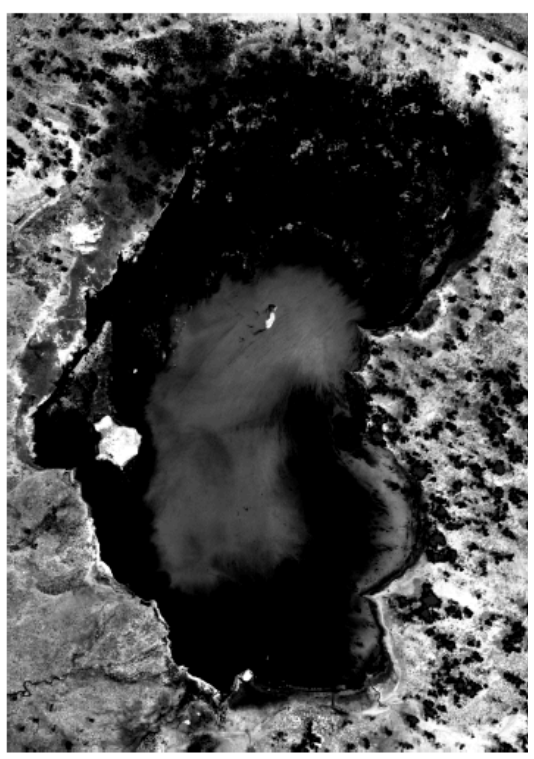

(b) 2013-01-17 NDVI map

Figure 2 - Close-up view of Lake Chilwa in the NDVI maps: (a) UK-DMC2 acquisition of $3^{\text {rd }}$ January 2013; (b) UK-DMC2 acquisition of $17^{\text {th }}$ January 2013.

This has been accomplished but not all the data have been returned yet to perform a comparison analysis. The next steps will be to correlate the results on quality of the water samples and the answers to the questionnaires submitted by the children, both collected in a crowd-sourcing experiment during the satellite imagery acquisition, with the imagery itself. 


\section{ACKNOWLEDGEMENTS}

The authors thank the Engineering and Physical Science Research Council (EPSRC) and the MILES (Models and Mathematics in Life and Social Sciences) programme at University of Surrey for funding this project.

\section{REFERENCES}

[1] www.wateraid.org

[2] Ritchie, J.C., P.V.Zimba, J.H.Everitt, "Remote Sensing Techniques to Assess Water Quality", Photogrammetric Engineering and Remote Sensing, vol. 69, no. 6, pp. 695-704, 2003.

[3] Kutser, T., "Passive optical remote sensing of cyanobacteria and other intense phytoplankton blooms in coastal and inland waters", International Journal of Remote Sensing, vol. 30, no. 17, pp. 4401-4425, 2009.

[4] Lillesand, T.M., Kiefer, R.W., Chipman J., Remote Sensing and Image interpretation, 6th edition, John Wiley \& Sons, 2008.

[5] Lin Q., Zhang Y., Nie Y., Guan Y., "'Detection of harmful algal blooms over the Gulf of Bohai sea in China at visible and near infrared (NIR) wavelengths of remote sensing", Journal of Electromagnetic Waves and Applications, vol.17, n.6, pp. 861-871, 2003. 